\title{
Polymerization in Carbone: A Novel Method for the Synthesis of More Sustainable Electrodes and Their Application as Cathodes for Lithium-Organic Energy Storage Materials Based On Vanillin
}

\author{
Ivan K. Ilic, Karen Leus, Johannes Schmidt, Jinyeon Hwang, Maria Maranska, Siegfried Eigler,
} and Clemens Liedel*

Cite This: ACS Sustainable Chem. Eng. 2020, 8, 3055-3064

Read Online

ABSTRACT: Sustainable energy storage materials are needed to implement necessary transitions to a more sustainable society. Therefore, we present novel vanillin (and thus ultimately possibly lignin)-derived electrode materials for lithium-ion-based energy storage systems. In the present approach, vanillin is first modified in two sustainable steps to afford bisvanillonitrile (BVN). The precursor materials for the electrodes are made from BVN and carbon black and are subsequently treated in the atmosphere of triflic acid in order to polymerize BVN. Used as a cathode material in a lithium-ion-based energy storage device, the resulting material shows capacities up to $90 \mathrm{mAh}^{-1}$ (respective to the whole electrode mass). This extraordinary performance can be attributed to a combination of non-Faradaic and Faradaic charge storage

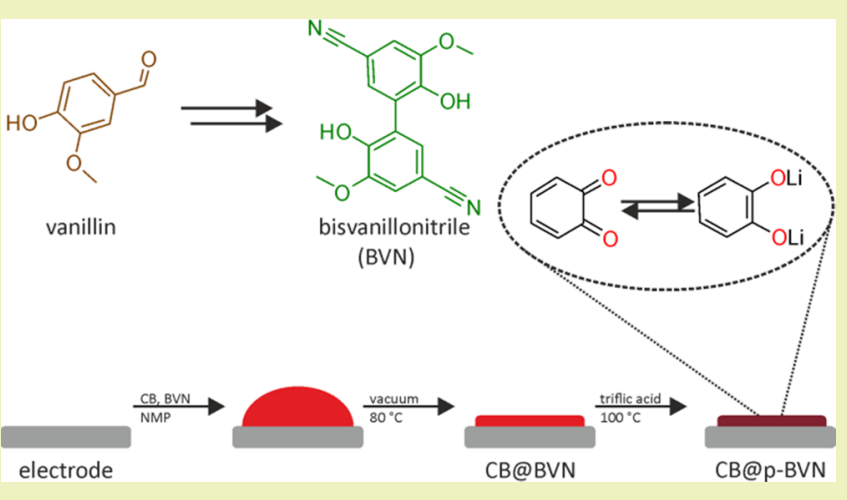
involving quinone units, which are abundantly found in the polymer backbone. In contrast to conventional organic electrode materials, excellent contact to carbon as a conductive additive is established by performing the polymerization in a mixture with carbon (in carbone), allowing the omission of additional unsustainable binder materials. Due to the sustainable synthesis and good performance, such sustainable electrodes may be applied in future energy storage devices.

KEYWORDS: energy storage, sustainable material, cross-linked polymer, hybrid material, triazine network

\section{INTRODUCTION}

In recent years, interest in sustainable sources of energy like solar, wind, or water power plants has been strongly increasing. In contrast, electrochemical energy storage devices are still based on unsustainable resources such as heavy-metal ores, and thus their replacement with more sustainable materials is required.

In this regard, electrodes derived from lignocellulosic biomass may be especially appealing. The first prominent biomass-based cathode for lithium-ion batteries included dilithium rhodizonate as an active component, ${ }^{2}$ a small molecule resulting in a low cyclability of the battery due to dissolution in the electrolyte. In attempts to reduce this solubility, polymeric species are advantageous, and naturally occurring polyphenols such as lignin ${ }^{3}$ were presented. Furthermore, synthetic polyphenols derived from sustainable phenols such as phenolic acids ${ }^{4}$ or guaiacol and syringol ${ }^{5}$ have been utilized. Similar bioderived polyphenols like polydopamine have been investigated for lithium- and sodium-ion batteries exhibiting promising results. ${ }^{6,7}$ However, dopamine cannot be obtained from natural resources on an industrially relevant scale, reducing the sustainability of polydopamine in batteries. Indeed, to the best of our knowledge, attempts to build a lithium-ion-based energy storage device using abundantly available biomaterials like sustainable polyphenols derived from lignocellulosic biomass without carbonization remain unsuccessful to this day.

Previously, we reported ChiVan, a compound created by grafting vanillin on chitosan using reductive amination. ${ }^{8}$ Although it showed remarkable properties for energy storage in an aqueous electrolyte, attempts to build a lithium-ion storage device remain unsuccessful. Vanillin-derived polymers without incorporation of chitosan may be more promising in this regard as they do not possess aliphatic hydroxyl groups. Plenty of other vanillin-based polymers have been reported so far, but only few of them keep the phenolic groups intact, which is a prerequisite for electrochemical activity. ${ }^{9}$ Polyesters $^{10}$ or polyimines ${ }^{11}$ made of dimerized bisvanillin and

Received: August 15, 2019

Revised: January 17, 2020

Published: February 10, 2020 
diacid or diamine species are an exception, and bisvanillin without any auxiliary species may also be polymerized by reductive coupling. ${ }^{12}$ However, on the one hand, the use of comonomers reduces the density of redox active functionalities and the sustainability if they are not biomass-based molecules themselves. On the other hand, to the best of our knowledge, attempts to synthesize a bisvanillin polymer by reductive coupling in large scales remain unsuccessful so far, making it unsuitable for development of sustainable materials.

Furthermore, regarding their sustainability, also the inactive components of electrodes need to be considered. Most polymeric active materials in energy storage devices exhibit low conductivity and are therefore always composited with conductive additives such as conductive polymers or carbons. However, conductive polymers are usually prepared from oilderived chemicals and are therefore not sustainable, ${ }^{13}$ unlike porous carbons that can be easily prepared from regrown biomass. ${ }^{14,15}$ In order to create a good composite with porous carbon, a polymer has to be soluble in the dispersing medium for carbon. Solubility in the electrolyte however is a common cause for shuttling of the polymer between the electrodes and subsequent self-discharge of the electrodes as well as low stability. Therefore, highly cross-linked, insoluble polymers are advantageous for battery applications, ${ }^{13}$ which hamper processability and contact to carbon materials. Besides the addition of binder polymers, cross-linking after forming a hybrid material with carbon may help to increase the stability and performance. ${ }^{16}$

For forming a highly cross-linked material, trimerization of aromatic polynitriles to create crystalline microporous polymers named covalent triazine frameworks (CTFs) has been first reported by Thomas et al. under high temperatures with zinc chloride as the catalyst. ${ }^{17}$ CTFs and related amorphous materials have been reported for many applications such as dye sorption, ${ }^{18}$ catalyst support, ${ }^{19}$ gas storage, ${ }^{20}$ and electrodevices exhibiting a high versatility. ${ }^{21-30}$ Later on, the low-temperature triflic acid-catalyzed polymerization of aromatic nitriles was used to obtain amorphous microporous polymers ${ }^{31,32}$ and to prepare hard-templated porous structures. ${ }^{33}$ Besides, also nonporous structures are available that showed remarkable properties for applications such as photocatalysis. $^{34}$

Herein, we aim to expand the polymerization of nitrilecontaining monomers to redox active materials. In order to prepare nonsoluble polymer electrodes that still can easily be processed in solution, we developed a new method inspired by the well-known dispersion polymerization approach and polymer/carbon nanotube hybrid materials in which the polymer is synthesized in the presence of nanotubes. ${ }^{6,35}$ This method, polymerization in carbone, utilizes casting of easily soluble monomers in a slurry with carbon onto the current collector as the first step. Subsequently, they are polymerized in the presence of the porous carbon matrix (in carbone) producing a highly cross-linked and insoluble polymer that is used as an active material in batteries. This method increases the contact between the polymer and the conductive carbons, facilitating the electron flow between the carbon and the polymer and consequently providing higher capacities. Specifically, we discuss the hybrid material of a vanillin-derived polymer that is formed via acid vapor-assisted solid-phase synthesis and carbon black as a sustainable lithium-organic energy storage device. The structure and electrochemical performance are discussed and compared to similar electrode compositions.

\section{EXPERIMENTAL SECTION}

2.1. Materials. Vanillin (Sigma-Aldrich), hydroxylammonium chloride (Sigma-Aldrich), iron(III) chloride (Fluka), magnesium sulfate (Acros Organics), triflic acid (TCI), sodium hydroxide (Sigma-Aldrich), 5-hydroxyisophthalonitrile (TCI), carbon black AB-520 (MTI Corporation), carbon paper Spectracarb 2050A-0550 (Fuel Cell Store), and the solvents acetic acid (VWR International), ethanol (Fischer), $\mathrm{N}$-methyl-2-pyrrolidone (NMP) (PanReac AppliChem), toluene (Fluka), diethyl ether (Fluka), and ammonia (25\%, Fluka) were used as received.

2.1.1. Synthesis of Vanillonitrile. ${ }^{36}$ Vanillin $(18.26 \mathrm{~g}, 120 \mathrm{mmol})$ and hydroxylammonium chloride $(12.50 \mathrm{~g}, 180 \mathrm{mmol})$ were dissolved in acetic acid $(100 \mathrm{~mL})$. The resulting mixture was heated under reflux conditions for $2 \mathrm{~h}$ with the formation of an orange solution. After cooling down, it was poured into diethyl ether $(300 \mathrm{~mL})$ and subsequently washed, once with water and twice with a $5 \%$ sodium hydroxide solution $(150 \mathrm{~mL}$ in each step). The aqueous phases were cleaned once with diethyl ether $(150 \mathrm{~mL})$. The organic phases were combined and dried with $\mathrm{MgSO}_{4}$. The solvent was removed under reduced pressure, and the product was recrystallized from toluene and dried under vacuum at $80{ }^{\circ} \mathrm{C}$ overnight. The procedure yielded 15.19 g (84.9\%) of pure vanillonitrile as confirmed by ${ }^{1} \mathrm{H}$ NMR and ${ }^{13} \mathrm{C}$ NMR.

${ }^{1} \mathrm{H}$ NMR (400 MHz, deuterated DMSO): $d=10.26 \mathrm{ppm}(\mathrm{s}, 1 \mathrm{H}$, $\mathrm{OH}), 7.35 \mathrm{ppm}(\mathrm{d}, 1 \mathrm{H}$, aromatic), $7.26 \mathrm{ppm}(\mathrm{m}, 1 \mathrm{H}$, aromatic), 6.89 ppm (d, $1 \mathrm{H}$, aromatic), $3.81 \mathrm{ppm}\left(\mathrm{s}, 3 \mathrm{H},-\mathrm{CH}_{3}\right)$.

${ }^{13} \mathrm{C}$ NMR (400 MHz, deuterated DMSO): $d=55.94 \mathrm{ppm}$ (methyl), $100.94 \mathrm{ppm}$ (aromatic), $115.24 \mathrm{ppm}$ (aromatic), $116.09 \mathrm{ppm}$ (aromatic), $119.67 \mathrm{ppm}$ (nitrile), $126.50 \mathrm{ppm}$ (aromatic), 147.98 ppm (aromatic), $151.46 \mathrm{ppm}$ (aromatic).

2.1.2. Synthesis of Bisvanillonitrile (BVN). ${ }^{37,38}$ Vanillonitrile $(10 \mathrm{~g}$, $50.3 \mathrm{mmol})$ was mixed with water $(240 \mathrm{~mL})$, and a solution of iron(III) chloride $(21.75 \mathrm{~g}, 100.6 \mathrm{mmol})$ in water $(240 \mathrm{~mL})$ was added. The mixture was heated under reflux conditions for $4 \mathrm{~h}$ and filtered afterward. The precipitate was collected and purified using Soxhlet extraction overnight with water as the purification solvent. The product was further cleaned with ethanol (three times centrifugation) and dried under vacuum at $80{ }^{\circ} \mathrm{C}$ overnight. The procedure yielded $4.77 \mathrm{~g}(48.0 \%)$ of pure bisvanillonitrile as confirmed by ${ }^{1} \mathrm{H}$ NMR, ${ }^{13} \mathrm{C}$ NMR, and MS, and a low iron content of 5.19 (SD, 0.082) $\mathrm{mg} \mathrm{g}^{-1}$ was confirmed with ICP-MS.

${ }^{1} \mathrm{H}$ NMR (400 MHz, deuterated DMSO): $d=9.86 \mathrm{ppm}(\mathrm{s}, 2 \mathrm{H}$, $\mathrm{OH}), 7.42 \mathrm{ppm}(\mathrm{s}, 2 \mathrm{H}$, aromatic), $7.23 \mathrm{ppm}(\mathrm{s}, 2 \mathrm{H}$, aromatic), 3.89 ppm $\left(\mathrm{s}, 6 \mathrm{H},-\mathrm{CH}_{3}\right)$.

${ }^{13} \mathrm{C}$ NMR $(400 \mathrm{MHz}$, deuterated DMSO): $d=56.39 \mathrm{ppm}$ (methyl), $100.35 \mathrm{ppm}$ (aromatic), $114.08 \mathrm{ppm}$ (aromatic), 119.56 ppm (nitrile), $124.61 \mathrm{ppm}$ (aromatic), $128.14 \mathrm{ppm}$ (aromatic), $147.94 \mathrm{ppm}$ (aromatic), $148.88 \mathrm{ppm}$ (aromatic).

MS (solvent: ACN, eluent: methanol/isopropanol (80/20) with $0.1 \%$ formic acid): calculated: 296.08 , measured: 296.0 (negative-ion mode).

2.1.3. Synthesis of $p-B V N .^{33}$ A vial $(10 \mathrm{~mL})$ containing BVN (50 $\mathrm{mg}$ ) was placed into a Schlenk tube (Figure S6 in the Supporting Information) with a volume of $\sim 120 \mathrm{~mL}$. The tube was evacuated, and $100 \mathrm{~mL}$ of argon was added to it followed by $0.6 \mathrm{~mL}$ of triflic acid. The tube was heated to $100{ }^{\circ} \mathrm{C}$ for $22 \mathrm{~h}$. Hereafter, the product was immersed in a $25 \%$ aqueous solution of ammonia for $1 \mathrm{~h}$. Afterward, the resulting polymer was centrifuged, washed with water and ethanol (two times, $50 \mathrm{~mL}$ of each solvent, $4000 \mathrm{rpm}, 3 \mathrm{~min}$ ), and dried under vacuum at $80{ }^{\circ} \mathrm{C}$ for $3 \mathrm{~h}$.

2.1.4. Synthesis of CB@p-BVN. ${ }^{33}$ Carbon black $(30 \mathrm{mg})$ and BVN $(30 \mathrm{mg})$ were put into a stainless steel jar $(12 \mathrm{~mL})$ and ground at 650 rpm for 50 min using a planetary ball mill PM100 (Retsch). Hereafter, NMP $(1 \mathrm{~mL})$ was added before further grinding for $10 \mathrm{~min}$. The obtained slurry $(40 \mu \mathrm{L})$ was spread across a circular piece of carbon paper (11 mm in diameter). The electrodes were dried under vacuum 
for $17 \mathrm{~h}$ at $80{ }^{\circ} \mathrm{C}$ before being placed inside a vial $(5 \mathrm{~mL})$. Two vials were put into a Schlenk tube with a volume of $\sim 120 \mathrm{~mL}$. The tube was evacuated, and $100 \mathrm{~mL}$ of argon was added to it followed by 0.6 $\mathrm{mL}$ of triflic acid. The tube was heated to $100{ }^{\circ} \mathrm{C}$ for $22 \mathrm{~h}$. The electrodes were subsequently immersed into a $25 \%$ aqueous solution of ammonia, water, and ethanol for $1 \mathrm{~h}$ each. Finally, they were dried under vacuum at $80{ }^{\circ} \mathrm{C}$ for $3 \mathrm{~h}$.

2.2. Morphology Characterization. The surface morphology was investigated by means of scanning electron microscopy (SEM) using a Zeiss Leo Gemini 1550 microscope.

2.3. Physisorption. Nitrogen physisorption measurements were performed on a Quantachrome Quadrasorb SI porosimeter at $77 \mathrm{~K}$.

2.4. Spectroscopy. The samples for ${ }^{1} \mathrm{H}$ and ${ }^{13} \mathrm{C}$ NMR were prepared by dissolving the material $(10 \mathrm{mg})$ in DMSO $\left(d_{6}, 0.65 \mathrm{~mL}\right)$. The spectra were measured using an Ascend $400 \mathrm{MHz}$ NMR spectrometer (Bruker). Fourier transform infrared (FT-IR) measurements were performed using a Nicolet iS 5 FT-IR Spectrometer (ThermoFisher Scientific). ${ }^{13} \mathrm{C}$ solid-state nuclear magnetic resonance (ss-NMR) spectrum was measured at static magnetic field $14.1 \mathrm{~T}$ (600 MHZ) with a JEOL JNM-ECZ600R/M1 spectrometer (Japan). $\mathrm{A}^{13} \mathrm{C}$ single-pulsed NMR experiment was performed at a MAS rate of $16 \mathrm{kHz}$ using a $\mathrm{ZrO}_{2} 3.2 \mathrm{~mm}$ MAS probe. The temperature of variable temperature cooling air was set to $275 \mathrm{~K}$ at a flow rate of 1070-1335 $\mathrm{L} / \mathrm{h}$. A nominal pulse angle of $90^{\circ}$, a pulse length of $2.13 \mu \mathrm{s}$, direct pulse, no $\mathrm{CP}$, no decoupling were used. The ${ }^{13} \mathrm{C}$ measurement ran with a relax delay of $300 \mathrm{~s}$ over 100 scans. The spectrum was referenced to external tetramethylsilane at $0 \mathrm{ppm}$ as a secondary reference. Inductively coupled plasma-optical emission spectroscopy (ICP-OES) was conducted using a Horiba Ultra 2 instrument equipped with photomultiplier tube detection. The samples were dissolved in aqua regia and filtered prior to analysis. X-ray photoelectron spectroscopy (XPS) measurements were performed using a ThermoScientific K-Alpha ${ }^{+} \mathrm{X}$-ray Photoelectron Spectrometer. All samples were analyzed using a microfocused, monochromated Al K $\alpha$ X-ray source (1486.68 eV; $400 \mu \mathrm{m}$ spot size). To prevent any localized charge buildup during analysis, the $\mathrm{K}$-Alpha ${ }^{+}$ charge compensation system was employed at all measurements. The samples were mounted on conductive carbon tape, and the resulting spectra were analyzed using the Avantage software from ThermoScientific.

2.5. Electrochemical Measurements. All electrochemical measurements were performed in Swagelok-type cells and tested using BioLogic MPG2 potentiostats. Lithium foil (Celgard 2325; 13 $\mathrm{mm}$ in diameter, $25 \mu \mathrm{m}$ thick), $1 \mathrm{M} \mathrm{LiPF}_{6}$ in ethylene carbonate, and diethyl carbonate (volumetric ratio of $1: 1,100 \mu \mathrm{L}$, solution used as obtained from Sigma-Aldrich) were used as a counter electrode, membrane, and electrolyte, respectively, and a circular carbon electrode with the active material on top was used as the working electrode. The electrolyte was chosen as it is one of the most common choices for carbonyl-based organic lithium-ion batteries, along with similar carbonyl electrolytes. ${ }^{39}$ All Swagelok-type cells were assembled in a glovebox with low water and oxygen levels. Charging-discharging measurements were performed at constant current density $\left(0.2 \mathrm{~A} \mathrm{~g}^{-1}\right.$ for 300 cycles) or at varying current density $\left(20\right.$ cycles at $0.05 \mathrm{~A} \mathrm{~g}^{-1}$, 10 cycles at $0.10 \mathrm{~A} \mathrm{~g}^{-1}, 10$ cycles at $0.20 \mathrm{~A} \mathrm{~g}^{-1}, 10$ cycles at $0.40 \mathrm{~A} \mathrm{~g}^{-1}$, and 10 cycles at $\left.0.80 \mathrm{~A} \mathrm{~g}^{-1}\right)$. In the displayed charging-discharging curves, the 15th (for $0.05 \mathrm{~A} \mathrm{~g}^{-1}$ ) or 5th cycle (for the other current densities) is shown. Cycling voltammetry was performed by cycling at $25 \mathrm{mV} \mathrm{s}^{-1}$ during 100 cycles and at $20 \mathrm{mV} \mathrm{s}^{-1}, 15 \mathrm{mV} \mathrm{s}^{-1}, 10 \mathrm{mV} \mathrm{s}^{-1}$, and $5 \mathrm{mV} \mathrm{s}^{-1}$ for five cycles each. In the displayed cycling voltammetry curves, always the last cycle of the respective rate is shown unless mentioned otherwise.

\section{RESULTS AND DISCUSSION}

3.1. Synthesis of BVN. Bisvanillonitrile (BVN) was obtained from vanillin in two steps using rather sustainable reaction conditions and media (Scheme S1). The first step, which concerns the substitution of the aldehyde with a nitrile group, was performed according to the previously reported procedure by Thompson et $\mathrm{al}^{36}$ In the second step, the dimerization of vanillonitrile, an oxidative coupling reaction mediated by iron(III) chloride, was used. ${ }^{37,38}$ As iron cations may be strongly chelated by BVN, the product was extensively purified using Soxhlet extraction with water, leaving a negligible iron contamination in the sample $\left(5.19 \mathrm{mg} \mathrm{g}^{-1}\right)$. The structure was confirmed by ${ }^{1} \mathrm{H}$ and ${ }^{13} \mathrm{C}$ NMR as well as MS (see the Experimental Section and Supporting Information).

3.2. Synthesis of $\boldsymbol{p}$-BVN. BVN was polymerized via an acid vapor-assisted solid-phase synthesis. ${ }^{33}$ The resulting polymer $(p-\mathrm{BVN})$ was characterized by means of FT-IR, ssNMR, and XPS.

When comparing the IR spectra of BVN and $p$-BVN (Figure la), the vibration at $3295 \mathrm{~cm}^{-1}$ (a), which corresponds to the
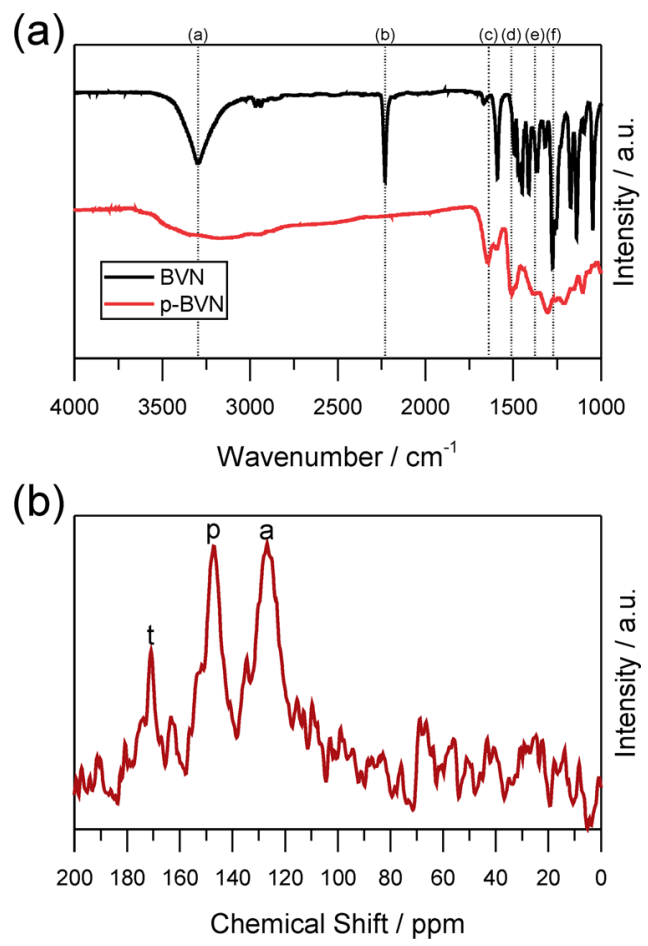

Figure 1. (a) FT-IR spectra of BVN and $p$-BVN. (b) Solid-state ${ }^{13} \mathrm{C}$ NMR spectrum of $p$-BVN.

hydroxyl vibrations, reduces in intensity and broadens after polymerization. Additionally, this peak could also partially correspond to the nitrogen-hydrogen bond vibration formed during hydrolysis of nitrile groups, as these two peaks would overlap. Furthermore, a sharp band at $2230 \mathrm{~cm}^{-1}$ (b), which can be assigned to the nitrile groups, is present in BVN but disappears during the reaction. As suggested in the literature, ${ }^{33}$ this observation points to the important role of nitrile groups in the polymerization of BVN. A sharp band at $1640 \mathrm{~cm}^{-1}$ (c), which appears in the spectrum after the polymerization, points to the formation of carbonyl groups either in the form of amide groups upon hydrolysis of nitrile groups or as part of oxidized hydroxyl groups. Two peaks at 1510 (d) and $1375 \mathrm{~cm}^{-1}$ (e) correspond to aromatic carbon-nitrogen vibrations, which is characteristic for triazine rings. ${ }^{23,33}$ Furthermore, a sharp vibration at $1270 \mathrm{~cm}^{-1}$ (f), which almost completely disappears after polymerization, can be ascribed to methoxy groups and indicates demethylation upon polymerization. $33,39,40$ 
(a)

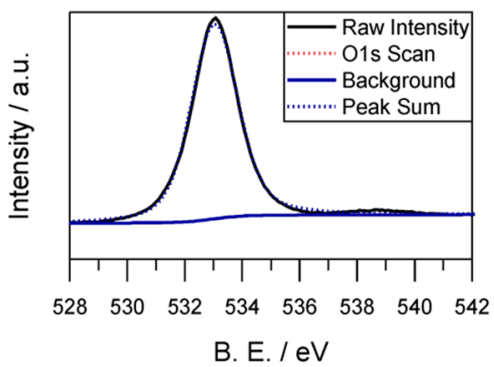

(d)

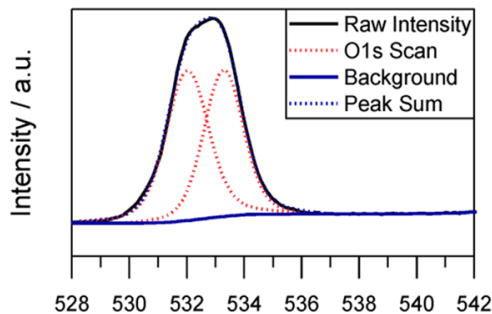

(b)

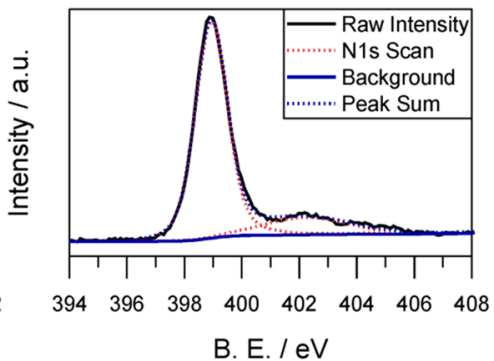

(e)

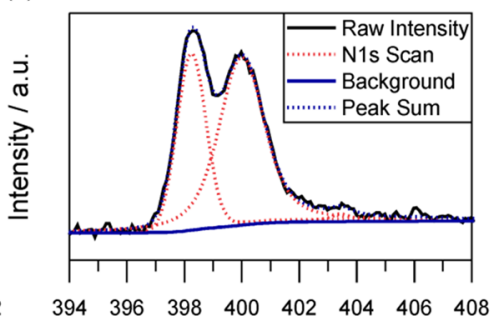

(c)

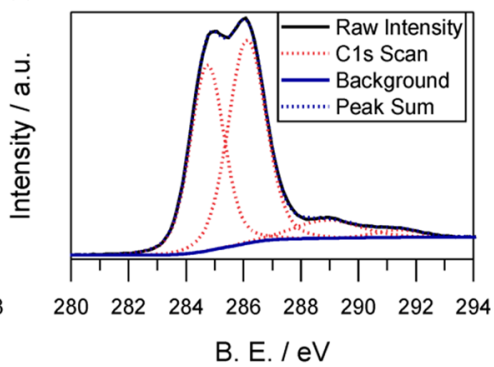

(f)

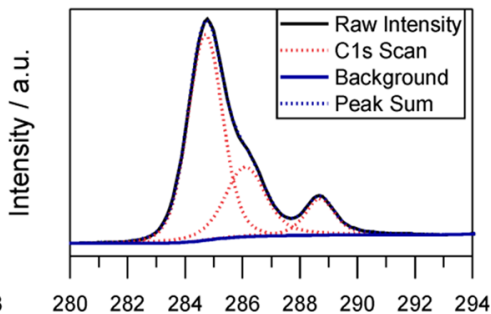

Figure 2. XPS spectra of $(\mathrm{a}-\mathrm{c}) \mathrm{BVN}$ and $(\mathrm{d}-\mathrm{f}) \mathrm{p}$-BVN. (a and $\mathrm{d}$ ) $\mathrm{O} 1 \mathrm{~s}$, (b and e) N 1s, and (c and f) C 1s.

The ${ }^{13} \mathrm{C}$ ss-NMR spectrum (Figure $1 \mathrm{~b}$ ) shows three signals in the range of 128,147 , and $170 \mathrm{ppm}$ that can be assigned to aromatic carbons (a), the $o$-hydroquinone unit $(\mathrm{p}),^{41}$ and triazine carbons $(\mathrm{t}),{ }^{42}$ respectively. In contrast to the (starting) reference material BVN, no peak at $56 \mathrm{ppm}$ can be identified, indicating cleavage of the methyl group in the methoxy unit. Appearance of triazine carbons and cleavage of methyl groups strongly support the successful polymerization. Noteworthy, no clear peaks that would denote quinones or amides are observed. These usually appear at higher chemical shift and may overlap with the triazine peak. ${ }^{41}$

In order to further investigate the polymerization mechanism, we performed XPS measurements of the individual compounds. In the energy range corresponding to oxygen 1s orbitals (Figure 2a), BVN has one observable peak at 533.04 $\mathrm{eV}$, which can be ascribed to oxygen that is bound to carbon via a single bond. After polymerization, two peaks with a similar area appear, one at $533.28 \mathrm{eV}$, which can be ascribed to the aforementioned oxygen species, and the second at 532.02 $\mathrm{eV}$, which can be ascribed to oxygen being part of a carbon= oxygen double bond, suggesting partial oxidation of the sample during polymerization. As discussed above, such bonds can be formed either as amide groups or as quinones. We want to emphasize that the samples were not treated with any carbonyl-containing solvent that might falsify the carbonyl signal at $532 \mathrm{eV}$.

In the energy range corresponding to nitrogen $1 \mathrm{~s}$ orbitals (Figure 2b), BVN has two observable peaks. The most dominant one at $398.94 \mathrm{eV}$ is characteristic for nitrogen in nitrile groups. A lower second peak at $402.24 \mathrm{eV}$ is a satellite peak, which is observable in molecules that have conjugated electron donors (hydroxyl and methoxyl groups) and acceptors (nitrile group). While this low intensity peak is still present after polymerization, the main peak is completely transformed into two new peaks of similar size. The peaks at 398.24 and $399.99 \mathrm{eV}$ can be ascribed to nitrogen in triazine and amide groups, respectively. These groups have been previously reported upon treatment of nitriles with triflic acid. ${ }^{31}$ However, as no significant amide peak was observed in ss-NMR, we conclude that amide formation is mainly a surface phenomenon.

In the energy range corresponding to carbon 1s orbitals (Figure 2c), BVN shows two dominant peaks and two peaks of low intensity. The dominant peaks at 284.74 and at $286.13 \mathrm{eV}$ can be ascribed to carbon bound to other carbon atoms and oxygen/nitrogen heteroatoms, respectively. Two weak peaks at 288.92 and $291.4 \mathrm{eV}$ indicate oxidized carbon and $\pi-\pi$ interactions, respectively. After polymerization, the ratio of the first two peaks changes so that the peak at $284.7 \mathrm{eV}$ is significantly more intense than the peak at $286.1 \mathrm{eV}$. This effect may indicate demethylation of the methoxy groups present in BVN during the course of oxidation upon treatment with triflic acid, that is, loss of a heteroatom-bound carbon. The peak representing the oxidized carbon indeed gets significantly more intense during the polymerization process, supporting the assumption of quinone formation in some of the aromatic groups, as discussed above. It is important to note though that the intensity of the carbon peaks in XPS analysis is often falsified by omnipresent carbon impurities.

Elemental analysis additionally confirms the demethylation of the methoxy functionalities upon acid vapor-assisted solidphase synthesis as the carbon content significantly decreases when comparing BVN and $p$-BVN and partial hydrolysis of nitrile groups as the oxygen content increases when comparing BVN and $p$-BVN (Table S1 in the Supporting Information).

In summary, we suggest that BVN mainly polymerizes via trimerization of nitrile groups followed by demethylation. Side reactions include hydrolysis of nitrile groups and oxidation during o-quinone formation. Such side products are most prominently observable in XPS analysis, indicating that they appear mostly at the surface (XPS is a surface technique).

3.3. Synthesis of $C B @ p-B V N$. In order to increase the conductivity of the $p$-BVN, we prepared a hybrid material with conductive carbon black (CB). For establishing good contact between the active material and carbon, we first made a slurry of carbon and BVN, casted the slurry on the current collector, dried it under vacuum, and subsequently performed the synthesis of $p$-BVN in the presence of carbon black (in carbone) in the same way as described above. The products 
after drying and after triflic acid treatment are denoted as $\mathrm{CB} @$ BVN and CB@p-BVN, respectively. This sequence of production steps makes later grinding of the hybrid material and blending with binders unnecessary. Scheme S2 shows the process.

In Figure 3, we compare the nitrogen sorption isotherms of the product ( $\mathrm{CB} @ p-\mathrm{BVN})$ to $\mathrm{CB}$ after ball milling with NMP

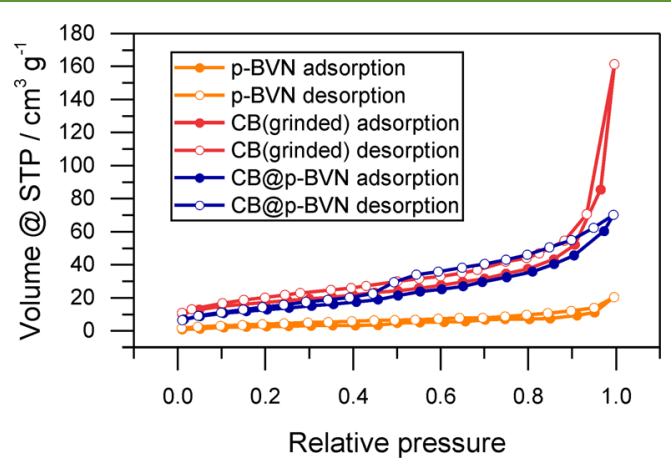

Figure 3. Nitrogen sorption isotherms of $p$-BVN (orange), CB(grinded) (red), and CB@p-BVN (blue).

( $\mathrm{CB}$ (grinded)) and $p$-BVN. $\mathrm{CB}$ (grinded) shows the highest BET surface area $\left(61.2 \mathrm{~m}^{2} \mathrm{~g}^{-1}\right)$. The isotherm indicates the presence of a small amount of micropores and a high external surface area. The significant reduction of the apparent surface area compared to CB before grinding (Figure S4) is ascribed to the presence of NMP during the grinding process, which blocks the majority of the micropores during the nitrogen sorption measurements due to its high boiling point. The BET surface area significantly drops during the $\mathrm{CB} @ p-\mathrm{BVN}$ fabrication $\left(46.7 \mathrm{~m}^{2} \mathrm{~g}^{-1}\right)$ due to the incorporation of nonporous $p$-BVN. The incorporation of the polymer not only results in additional blocking of some micropores but also especially holds carbon black particles together, reducing the external surface area. Nevertheless, the isotherm has a similar shape as that of $\mathrm{CB}$ (grinded) and indicates both micropores and a significant contribution of the external surface area to nitrogen sorption.
SEM analysis provides further insights into the structure formation. As can be seen from Figure 4, $\mathrm{CB}$ (grinded) (without the addition of any binder material) shows a grainy structure characteristic for carbon black. Upon the addition of BVN, rod/platelet-like particles appear between the grains, which can be identified as particles of BVN. Upon polymerization, these structures disappear, and instead, the grainy carbon black is covered with a web-like structure that can be ascribed to $p-\mathrm{BVN}$, indicating good contact between $\mathrm{CB}$ and $p$ BVN.

XPS measurements of the hybrid materials confirm the formation of the same structures as discussed in the pristine organic material (Figure S5 in the Supporting Information). The oxygen 1 s peak at $532.22 \mathrm{eV}$ in the sample of carbon black corresponds to oxygen on the carbon surface, which was introduced as a consequence of ball milling. ${ }^{43}$ In the $\mathrm{CB} @$ BVN and CB@p-BVN materials, this peak is also observed but overlaps with the above described peaks of BVN and $p$-BVN. A significantly increased intensity of the carbonyl oxygen peak of $\mathrm{CB} @ p-\mathrm{BVN}$ in comparison to $\mathrm{CB} @ \mathrm{BVN}$ supports the formation of quinones and amides in some of the aromatic and nitrile groups, respectively, during acid vapor-assisted solid-phase synthesis. Nitrogen on the carbon surface introduced from NMP during ball milling is observed in the energy range corresponding to the nitrogen 1 s orbital at 400.32 $\mathrm{eV}$. Also here, the observed peaks conform to the samples without carbon black. Carbon black finally shows three dominant peaks at 284.67, 285.55, and $288.22 \mathrm{eV}$ in the energy range corresponding to the carbon $1 \mathrm{~s}$ orbital, which also dominate the carbon 1s spectra of CB@BVN and CB@p$\mathrm{BVN}$, overlapping with the above indicated peaks of BVN and $p$-BVN.

Furthermore, thermogravimetric analysis was carried out (see Figure S7 in the Supporting Information). It was observed that upon grinding, the thermal stability of carbon black is reduced, which can be ascribed to the high oxygen content in $\mathrm{CB}$ (grinded) (Table S1 in the Supporting Information) that is a consequence of the modification of carbon upon ball milling. The TGA curve of CB@p-BVN can be seen as a combination of $\mathrm{CB}$ (grinded) and $p$-BVN and does not show a significant weight loss in the range of $400{ }^{\circ} \mathrm{C}$ (decomposition

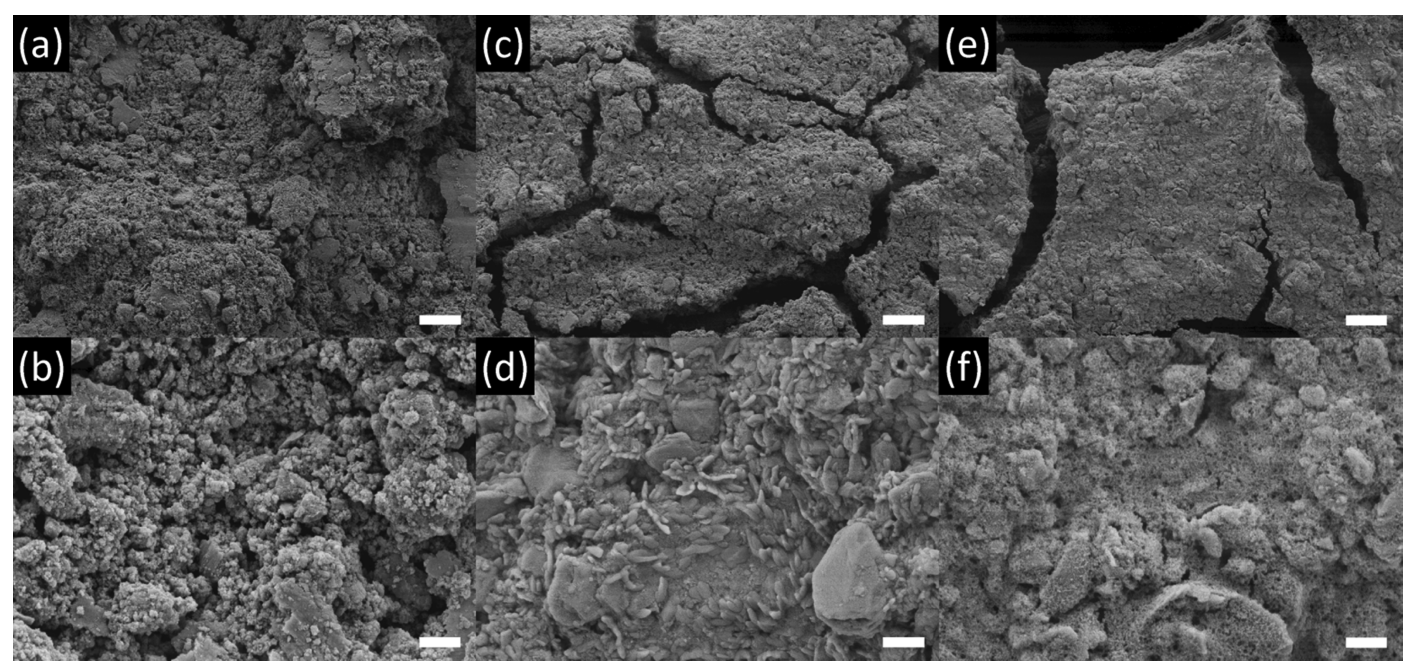

Figure 4. SEM images of (a and b) CB(grinded), (c and d) CB@BVN, and (e and f) CB@p-BVN. The scale bars represent (a, c, and e) $10 \mu \mathrm{m}$ and (b, d, and f) $1 \mu \mathrm{m}$. 
temperature of BVN). This observation confirms the polymerization of BVN in the presence of carbon black and the formation of a hybrid material of $\mathrm{CB}$ and $p$-BVN.

3.4. Energy Storage of CB@p-BVN. Cyclic voltammetry of $\mathrm{CB} @ p-\mathrm{BVN}$ initially shows very weak peaks (Figure S8). However, with prolonged cycling, an oxidation peak around $3.7 \mathrm{~V}$ and a reduction peak around $2.8 \mathrm{~V}$ become visible. The increasing intensity of the redox couple peak and high overpotential can be explained by the necessary migration of $\mathrm{Li}^{+}$cations and bulky $\mathrm{PF}_{6}^{-}$anions inside the electrode, resulting in a rearrangement of the CB@p-BVN hybrid material. Furthermore, before $p$-BVN can contribute to the capacity by reversible redox reactions, its $o$-hydroquinone moieties (and possible quinone moieties formed upon polymerization as described above) have to be lithiated (Scheme S3). Cycling CB@p-BVN at different sweep rates clearly denotes semireversible behavior (Figure S8b). We further use electrodes without $p$-BVN to estimate the contribution of $p-\mathrm{BVN}$ to the overall capacity of the CB@p$\mathrm{BVN}$ electrodes. These $p$-BVN-free electrodes (further denoted as $\mathrm{CB}$ (trif)) contain only carbon black (and $10 \%$ of PVDF as a binder for stabilization) and are treated in the same way as the CB@p-BVN electrodes for better comparison, that is, are also exposed to triflic acid during electrode formation. Such CB(trif) electrodes however show significantly lower performance in cyclic voltammetry without any redox peaks in the cyclic voltammograms (Figure 5a), demonstrating the importance of $p$-BVN for a reversible redox behavior of the electrodes. Furthermore, the first discharging cycle of the preformed material (Figure S9) starts at 3.2 V (the open circuit potential), confirming partial oxidation of hydroquinone moieties during synthesis.

Charging and discharging of CB@p-BVN (Figure 5b and Figure S9a) at different current densities shows belly-shaped galvanostatic discharging curves, which are characteristic for devices that store charges in a combination of battery- and capacitor-like reactions. ${ }^{44}$ This behavior is often incorrectly described in the literature as pseudocapacitance. ${ }^{45,46}$ The slope of the discharging curves is the lowest around $3 \mathrm{~V}$, which corresponds well to the discharging peak in cyclic voltammograms. In contrast, $\mathrm{CB}$ (trif) shows triangular galvanostatic charging-discharging curves, which indicate purely capacitorlike charge storage. The capacity of $\mathrm{CB}$ (trif) is $38.7 \mathrm{mAh} \mathrm{g}^{-1}$ compared to $85.2 \mathrm{mAh} \mathrm{g}^{-1}$ for CB@p-BVN (fifth cycle, $0.05 \mathrm{~A}$ $\mathrm{g}^{-1}$ ), which proves that $p$-BVN is mainly responsible for charge storage in $\mathrm{CB} @ p-\mathrm{BVN}$, in which p-BVN contributes primarily to Farradaic charge storage, while $\mathrm{CB}$ additionally contributes primarily to non-Farradaic charge storage.

In the past, triazine rings were thought to significantly contribute to energy storage in CTFs and related materials used as cathodes in lithium-organic batteries, ${ }^{22,30}$ but this assumption was disproved later. ${ }^{29}$ Therefore, we conclude that the triazine rings in our material rather reduce the solubility of $p$-BVN by acting as cross-linking centers. As a mechanism of Faradaic charge storage in the case of CB@p-BVN, we instead suggest the reversible redox reaction between ortho-hydroquinone and ortho-quinone as shown for similar systems in aqueous $^{3,47,48}$ and organic lithium-containing electrolytes ${ }^{49}$ (Scheme S2).

In order to even more clearly demonstrate the significance of $p$-BVN for charge storage, we further exchanged $\mathrm{BVN}$ in the synthesis of CB@p-BVN by a similar compound, namely, 5hydroxyisophthalonitrile (HIT), which however does not
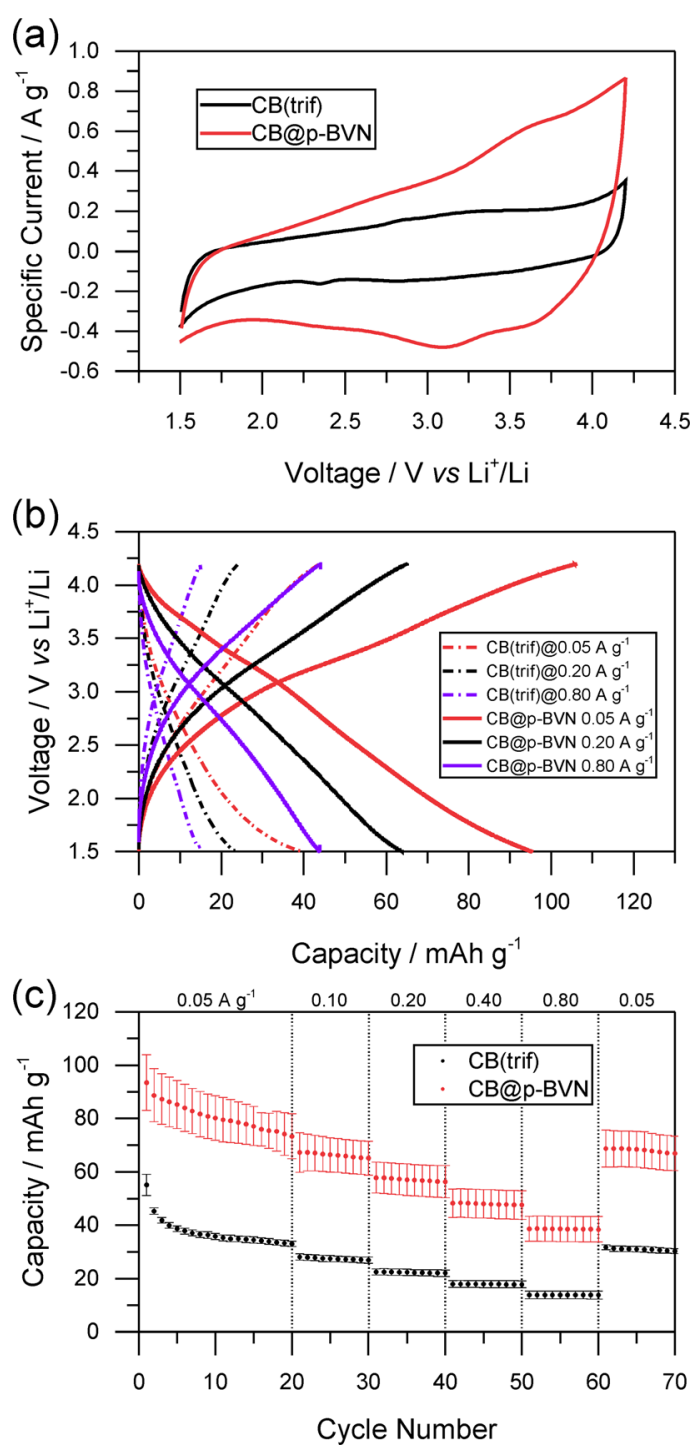

Figure 5. Electrochemical performance of $\mathrm{CB} @ p-\mathrm{BVN}$ and $\mathrm{CB}($ trif $)$ in a lithium half-cell setup with lithium as the counter electrode and 1 $\mathrm{M} \mathrm{LiPF}_{6}$ in EC/DEC $(1 / 1)$ as the electrolyte. (a) Cyclic voltammetry at $5 \mathrm{mV} \mathrm{s}^{-1}$. (b) Charging-discharging tests at selected current densities. (c) Discharging capacities as calculated from chargingdischarging tests at different current densities.

provide quinones for charge storage capability. CB@p-HIT was prepared analogous to CB@p-BVN. Charging-discharging tests at different current densities (Figure S11) clearly indicate significantly better performance of CB@p-BVN and the absence of a belly-shaped discharging behavior in CB@pHIT. The surplus capacity of $\mathrm{CB} @ p-\mathrm{BVN}$ when compared to $\mathrm{CB} @ p$-HIT in the range of $20 \mathrm{mAh} \mathrm{g}^{-1}$ clearly indicates the contribution of quinones for charge storage, albeit with significantly lower capacity than theoretically predicted. The deviation is likely caused by contribution of phenolic groups to cross-linking as discussed above, irreversible oxidation reactions, and limited accessibility of active groups to the electrolyte. Still, quinone groups in CB@p-BVN enhance the charge storage properties of this triazine-containing network significantly. Enhanced charge storage of CB@p-HIT when compared to $\mathrm{CB}$ (trif) may be explained by increased hydrophilicity ${ }^{43,50,51,52}$ upon introduction of heteroatoms to carbon. We further note that the capacity of CB@p-BVN is 
lower than the theoretical Faradaic capacity (Supporting Information). Besides the imperfect structure that clearly is not an ordered triazine framework, limited contact to the electrolyte and current collector may explain this behavior to some extent. Unfortunately, unexpectedly low capacities are common among organic electroactive materials, even when they have unambiguous structure, as is the case for crystalline materials. ${ }^{53}$ Optimization and detailed analysis will be the focus of future studies and described elsewhere.

Figure $5 c$ shows the rate capability of the CB@p-BVN electrodes, calculated from the charging and discharging curves at different current densities. An initial discharging capacity of $85.2 \mathrm{mAh} \mathrm{g}^{-1}$ at a moderate current density of $0.05 \mathrm{~A} \mathrm{~g}^{-1}$ decreases to 66.4, 57.1, 47.8, and $38.5 \mathrm{mAh} \mathrm{g}^{-1}$ at current densities of $0.1,0.2,0.4$, and $0.8 \mathrm{~A} \mathrm{~g}^{-1}$, respectively, and retains $80 \%$ of the initial capacity $\left(68.5 \mathrm{~A} \mathrm{~g}^{-1}\right)$ once the current density is restored to $0.05 \mathrm{~A} \mathrm{~g}^{-1}$ (capacities represent the fifth cycle at the respective current density). The decrease in the discharging capacity at $0.05 \mathrm{~A} \mathrm{~g}^{-1}$ within 60 cycles, some of them at higher current density, can be ascribed to the instability of the electrodes, for example, by over oxidation, as in general organic materials show a relatively low stability during electrochemical cycling as compared to inorganic electrodes.

Similarly, charging and discharging for 300 cycles at a constant current density of $0.2 \mathrm{~A} \mathrm{~g} \mathrm{~g}^{-1}$ results in a constant decrease in the discharging capacity with the exception of only the first six cycles, for which a slight increase is noted (Figure 6a). Capacity retention is relatively high, retaining $66.7 \%$ of the discharging capacity after 300 cycles. Comparing EIS before and after cycling (Figure S12) clearly shows that during cycling, new interfaces form. The Nyquist plots show a complex behavior with hints of multiple semicircles after cycling, denoting the degradation of structures, macroscopic rearrangements, and the loss of contact, as indicated by decreasing capacity. The exact mechanism of $p$-BVN degradation remains unclear. When comparing samples that were simply immersed in the electrolyte to samples that were charged and discharged for 300 cycles (Figure S13), a significant incorporation of fluorine at the expense of oxygen is apparent in cycled samples. Thus, energy-dispersive X-ray measurements indicate that oxygen is partly exchanged by fluorine from the electrolyte during cycling. Additionally, SEM images show a flatter surface in cycled samples probably due to volumetric changes upon cycling (Figure S13).

Finally, we emphasize the importance of the polymerization in carbone, that is, in the presence and in tight contact with carbon. In order to quantify the necessity of the polymerization process, we compare the $\mathrm{CB} @ p$-BVN electrodes to conventionally fabricated organic electrodes having the same active material and carbon support in the same ratio on the current collector with a similar loading. The capacity of these conventional electrodes in which a slurry of the active material $p$-BVN (45\%), carbon black (45\%), and PVDF as the binder $(10 \%)$ is prepared by means of ball milling is summarized in Figure $4 \mathrm{~b}$ (denoted as $\mathrm{CB}+p$-BVN + PVDF). The discharging capacity of $\mathrm{CB} @ p-\mathrm{BVN}$ grossly outperforms $\mathrm{CB}+p-\mathrm{BVN}+$ PVDF (Figure 6c). In early cycles, the improvement in the capacity by the polymerization in carbone fluctuates between 17 and $32 \%$ and soon stabilizes around $21 \%$. This magnitude greatly exceeds the expected improvement of $10 \%$ due to the omission of the binder and is explained by the better contact between the components after polymerization in carbone. We (a)

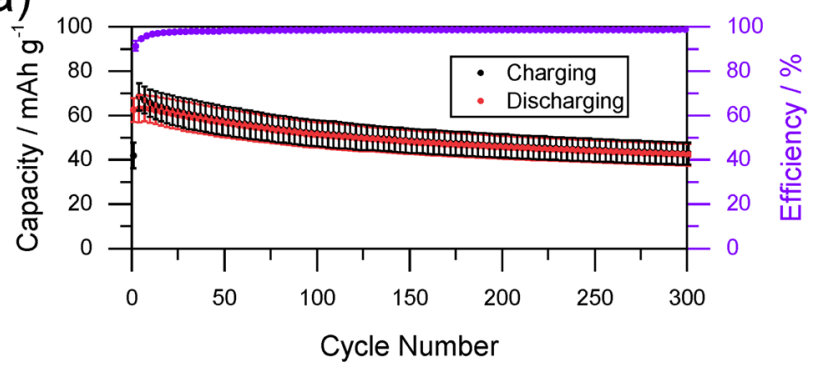

(b)

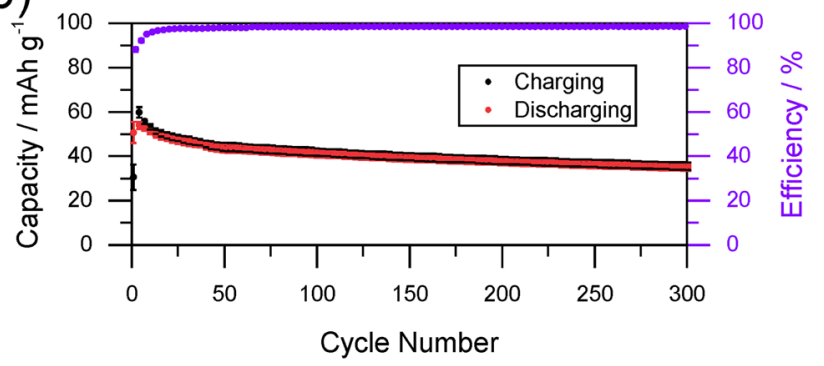

(c)

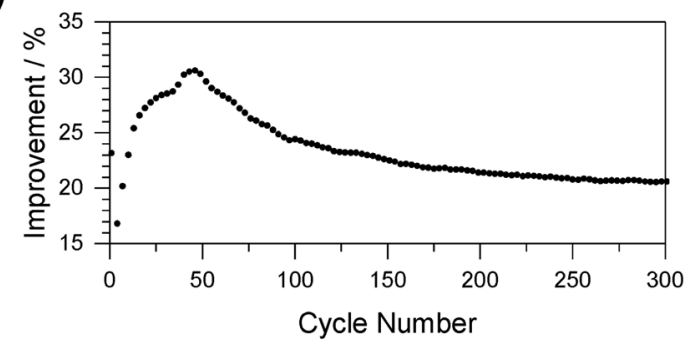

Figure 6. Electrochemical performance of $\mathrm{CB} @ p-\mathrm{BVN}$ and $\mathrm{CB}+p$ $\mathrm{BVN}+\mathrm{PVDF}$ in a lithium half-cell setup with lithium as the counter electrode and $1 \mathrm{M} \mathrm{LiPF}_{6}$ in EC/DEC $(1 / 1)$ as the electrolyte. Every third data point is displayed for clarity. (a) Capacity of CB@p-BVN at $0.2 \mathrm{~A} \mathrm{~g}^{-1}$ as calculated from charging-discharging tests. (b) Capacity of $\mathrm{CB}+p-\mathrm{BVN}+\mathrm{PVDF}$ at $0.2 \mathrm{~A} \mathrm{~g}^{-1}$ as calculated from chargingdischarging tests. (c) Comparison of the discharging capacities of $\mathrm{CB} @ p-\mathrm{BVN}$ and $\mathrm{CB}+p-\mathrm{BVN}+\mathrm{PVDF}$ in (a) and (b), respectively.

note that upon polymerization in carbone in the presence of the same binder, the resulting PVDF-containing electrodes (made of $45 \%$ BVN, $45 \%$ carbon black, and 10\% PVDF) behave similarly to CB@p-BVN (Figure S14). The presence of a binder is therefore neither important for stability reasons nor is the lower capacity in binder-containing electrodes after conventional manufacturing a result of the binder content. A significant increase in the capacity is observed due to in carbone polymerization, confirming the importance of the good contact between the polymer and the conductive additive. However, the final Faradaic capacity is still lower than the theoretically calculated one, suggesting that the contact between the polymer and the carbon black is still not optimal. In contrast to most other organic electrodes, however, the CB@p-BVN electrodes may easily be fabricated without any fluorinated chemicals, increasing the sustainability of the process while simultaneously increasing the capacity.

\section{CONCLUSIONS}

A novel cathode material derived from lignocellulosic biomass was presented. Specifically, vanillin, which can industrially be synthesized from lignin, was modified in two easy and sustainable steps affording bisvanillonitrile. The latter has a 
high density of redox active groups and two nitrile functionalities, allowing the formation of a highly cross-linked aromatic network upon trimerization of the nitrile groups in the presence of triflic acid. For the fabrication of the electrodes, a novel approach was developed, in which a composite of monomer and carbon black was first formed. Upon polymerization, tight interactions in the hybrid material were obtained. Not only did this method allow the omission of a binder as the redox active polymer is formed around the grains of carbon black and thus served as an efficient binder itself but it also allowed for a better contact between the carbon black and polymer, significantly improving the charge storage performance. To the best of our knowledge, the presented hybrid energy storage material is the first report of a lignin-derived cathode for lithium-ion-based energy storage and may hence contribute to more sustainable energy storage devices in the future.

\section{ASSOCIATED CONTENT}

\section{(3) Supporting Information}

The Supporting Information is available free of charge at https://pubs.acs.org/doi/10.1021/acssuschemeng.9b04797.

Schemes, NMR, mass spectroscopy, nitrogen physisorption, additional XPS, elemental analysis, TGA, additional $\mathrm{CV}$, additional charging-discharging curves, calculation of the theoretical capacity, and EIS data (PDF)

\section{AUTHOR INFORMATION}

\section{Corresponding Author}

Clemens Liedel - Department of Colloid Chemistry, Max Planck Institute of Colloids and Interfaces, 14476 Potsdam, Germany; (1) orcid.org/0000-0002-2323-9431;

Email: Clemens.Liedel@mpikg.mpg.de

\section{Authors}

Ivan K. Ilic - Department of Colloid Chemistry, Max Planck Institute of Colloids and Interfaces, 14476 Potsdam, Germany

Karen Leus - Center for Ordered Materials, Organometallics and Catalysis, Department of Chemistry, Ghent University, Ghent 9000, Belgium

Johannes Schmidt - Institute of Chemistry, Functional Materials, Technische Universität Berlin, 10623 Berlin, Germany

Jinyeon Hwang - Department of Colloid Chemistry, Max Planck Institute of Colloids and Interfaces, 14476 Potsdam, Germany

Maria Maranska - Institute of Chemistry and Biochemistry, Freie Universität Berlin, Berlin 14195, Germany

Siegfried Eigler - Institute of Chemistry and Biochemistry, Freie Universität Berlin, Berlin 14195, Germany; 이이.org/00000002-0536-8256

Complete contact information is available at: https://pubs.acs.org/10.1021/acssuschemeng.9b04797

\section{Author Contributions}

I.K.I., K.L., and C.L. designed the project. I.K.I, C.L., and J.H. designed the electrochemical measurements. I.K.I carried out the synthesis, infrared spectroscopy, nitrogen physisorption and electrochemical measurements. J.S. carried out XPS measurement and analysis. J.H. performed SEM measurements. M.M. and S.E. performed and evaluated solid-state NMR experiments. I.K.I. and C.L. wrote the manuscript. All authors have given approval to the final version of the manuscript.

\section{Funding}

We appreciate financial support by the German Research Foundation (DFG, grant no. LI 2526/4-1), the Max Planck Society, and the Max Planck Institute of Colloids and Interfaces. We would like to acknowledge the assistance of the Core Facility BioSupraMol supported by the DFG and the DFG with grant no. 392444269.

\section{Notes}

The authors declare no competing financial interest.

\section{ACKNOWLEDGMENTS}

We acknowledge the help in the laboratory and with ICP-MS measurements by Jessica Brandt. We further thank Michael J. Bojdys and Markus Antonietti for fruitful discussions; Milena Perovic for the help with physisorption measurements; Olaf Niemeyer, Steffen Tröger-Müller, and Alexandra Tsouka for the help with NMR measurements; Antje Völkel for the help with elemental analysis and TGA measurements; and to Ante Grkovic for coining the term "in carbone". The reviewers of our manuscript are appreciated for their constructive comments. Last, we would like to thank the Chez Briel-Institution for the substantial support.

\section{REFERENCES}

(1) Larcher, D.; Tarascon, J. M. Towards Greener and More Sustainable Batteries for Electrical Energy Storage. Nat. Chem. 2015, 7, 19-29.

(2) Chen, H.; Armand, M.; Demailly, G.; Dolhem, F.; Poizot, P.; Tarascon, J. M. From Biomass to a Renewable $\mathrm{LiXC}_{6} \mathrm{O}_{6}$ Organic Electrode for Sustainable Li-Ion Batteries. ChemSusChem 2008, 1, $348-355$.

(3) Milczarek, G.; Inganas, O. Renewable Cathode Materials from Biopolymer/Conjugated Polymer Interpenetrating Networks. Science 2012, 335, 1468-1471.

(4) Wagner, M.; Rębiś, T.; Inganäs, O. Enhancing Charge Storage of Conjugated Polymer Electrodes with Phenolic Acids. J. Power Sources 2016, 302, 324-330.

(5) Rębís, T.; Nilsson, T. Y.; Inganäs, O. Hybrid Materials from Organic Electronic Conductors and Synthetic-Lignin Models for Charge Storage Applications. J. Mater. Chem. A 2016, 4, 1931-1940.

(6) Liu, T.; Kim, K. C.; Lee, B.; Chen, Z.; Noda, S.; Jang, S. S.; Lee, S. W. Self-Polymerized Dopamine as an Organic Cathode for Li- and Na-Ion Batteries. Energy Environ. Sci. 2017, 10, 205-215.

(7) Liedel, C.; Wang, X.; Antonietti, M. Biobased Polymer Cathodes with Enhanced Charge Storage. Nano Energy 2018, 53, 536-543.

(8) Ilic, I. K.; Meurer, M.; Chaleawlert-umpon, S.; Antonietti, M.; Liedel, C. Vanillin Decorated Chitosan as Electrode Material for Sustainable Energy Storage. RSC Adv. 2019, 9, 4591-4598.

(9) Fache, M.; Boutevin, B.; Caillol, S. Vanillin, a Key-Intermediate of Biobased Polymers. Eur. Polym. J. 2015, 68, 488-502.

(10) Llevot, A.; Grau, E.; Carlotti, S.; Grelier, S.; Cramail, H. Renewable (Semi)aromatic Polyesters from Symmetrical VanillinBased Dimers. Polym. Chem. 2015, 6, 6058-6066.

(11) Amarasekara, A. S.; Razzaq, A. Vanillin-Based Polymers-part II: Synthesis of Schiff Base Polymers of Divanillin and Their Chelation with Metal Ions. ISRN Polym. Sci. 2012, 2012, 1-5.

(12) Amarasekara, A. S.; Wiredu, B.; Razzaq, A. Vanillin Based Polymers: I. An Electrochemical Route to Polyvanillin. Green Chem. 2012, 14, 2395-2397.

(13) Muench, S.; Wild, A.; Friebe, C.; Häupler, B.; Janoschka, T.; Schubert, U. S. Polymer-Based Organic Batteries. Chem. Rev. 2016, $116,9438-9484$.

(14) Berthold, T.; Castro, C. R.; Winter, M.; Hoerpel, G.; Kurttepeli, M.; Bals, S.; Antonietti, M.; Fechler, N. Tunable Nitrogen-Doped 
Carbon Nanoparticles from Tannic Acid and Urea and Their Potential for Sustainable Soots. ChemNanoMat 2017, 3, 311-318.

(15) Yan, R.; Antonietti, M.; Oschatz, M. Toward the Experimental Understanding of the Energy Storage Mechanism and Ion Dynamics in Ionic Liquid Based Supercapacitors. Adv. Energy Mater. 2018, 8, 112.

(16) Chaleawlert-umpon, S.; Liedel, C. More Sustainable Energy Storage: Lignin Based Electrodes with Glyoxal Crosslinking. J. Mater. Chem. A 2017, 5, 24344-24352.

(17) Kuhn, P.; Antonietti, M.; Thomas, A. Porous, Covalent Triazine-Based Frameworks Prepared by Ionothermal Synthesis. Angew. Chem., Int. Ed. 2008, 47, 3450-3453.

(18) Kuhn, P.; Krüger, K.; Thomas, A.; Antonietti, M. "Everything Is Surface": Tunable Polymer Organic Frameworks with Ultrahigh Dye Sorption Capacity. Chem. Commun. 2008, 44, 5815-5817.

(19) Palkovits, R.; Antonietti, M.; Kuhn, P.; Thomas, A.; Schüth, F. Solid Catalysts for the Selective Low-Temperature Oxidation of Methane to Methanol. Angew. Chem., Int Ed. 2009, 48, 6909-6912.

(20) Katekomol, P.; Roeser, J.; Bojdys, M.; Weber, J.; Thomas, A. Covalent Triazine Frameworks Prepared from 1,3,5-Tricyanobenzene. Chem. Mater. 2013, 25, 1542-1548.

(21) Hao, L.; Luo, B.; Li, X.; Jin, M.; Fang, Y.; Tang, Z.; Jia, Y.; Liang, M.; Thomas, A.; Yang, J.; et al. Terephthalonitrile-Derived Nitrogen-Rich Networks for High Performance Supercapacitors. Energy Environ. Sci. 2012, 5, 9747.

(22) Sakaushi, K.; Nickerl, G.; Wisser, F. M.; Nishio-Hamane, D.; Hosono, E.; Zhou, H.; Kaskel, S.; Eckert, J. An Energy Storage Principle Using Bipolar Porous Polymeric Frameworks. Angew. Chem, Int Ed. 2012, 51, 7850-7854.

(23) Sakaushi, K.; Hosono, E.; Nickerl, G.; Gemming, T.; Zhou, H.; Kaskel, S.; Eckert, J. Aromatic Porous-Honeycomb Electrodes for a Sodium-Organic Energy Storage Device. Nat. Commun. 2013, 4, 1485.

(24) Liao, H.; Ding, H.; Li, B.; Ai, X.; Wang, C. Covalent-Organic Frameworks: Potential Host Materials for Sulfur Impregnation in Lithium-sulfur Batteries. J. Mater. Chem. A 2014, 2, 8854-8858.

(25) Hao, L.; Ning, J.; Luo, B.; Wang, B.; Zhang, Y.; Tang, Z.; Yang, J.; Thomas, A.; Zhi, L. Structural Evolution of 2D Microporous Covalent Triazine-Based Framework toward the Study of HighPerformance Supercapacitors. J. Am. Chem. Soc. 2015, 137, 219-225.

(26) Li, Y.; Zheng, S.; Liu, X.; Li, P.; Sun, L.; Yang, R.; Wang, S.; Wu, Z. S.; Bao, X.; Deng, W. Q. Conductive Microporous Covalent Triazine-Based Framework for High-Performance Electrochemical Capacitive Energy Storage. Angew. Chem., Int. Ed. 2018, 7992-7996. (27) Talapaneni, S. N.; Hwang, T. H.; Je, S. H.; Buyukcakir, O.; Choi, J. W.; Coskun, A. Elemental-Sulfur-Mediated Facile Synthesis of a Covalent Triazine Framework for High-Performance Lithium-Sulfur Batteries. Angew. Chem., Int Ed. 2016, 55, 3106-3111.

(28) Je, S. H.; Kim, H. J.; Kim, J.; Choi, J. W.; Coskun, A. Perfluoroaryl-Elemental Sulfur $S_{N} A r$ Chemistry in Covalent Triazine Frameworks with High Sulfur Contents for Lithium-Sulfur Batteries. Adv. Funct. Mater. 2017, 27, 1703947.

(29) See, K. A.; Hug, S.; Schwinghammer, K.; Lumley, M. A.; Zheng, Y.; Nolt, J. M.; Stucky, G. D.; Wudl, F.; Lotsch, B. V.; Seshadri, R. Lithium Charge Storage Mechanisms of Cross-Linked Triazine Networks and Their Porous Carbon Derivatives. Chem. Mater. 2015, 27, 3821-3829.

(30) Sakaushi, K.; Hosono, E.; Nickerl, G.; Zhou, H.; Kaskel, S.; Eckert, J. Bipolar Porous Polymeric Frameworks for Low-Cost, HighPower, Long-Life All-Organic Energy Storage Devices. J. Power Sources 2014, 245, 553-556.

(31) Ren, S.; Bojdys, M. J.; Dawson, R.; Laybourn, A.; Khimyak, Y. Z.; Adams, D. J.; Cooper, A. I. Porous, Fluorescent, Covalent Triazine-Based Frameworks via Room-Temperature and MicrowaveAssisted Synthesis. Adv. Mater. 2012, 24, 2357-2361.

(32) Zhu, X.; Tian, C.; Mahurin, S. M.; Chai, S. H.; Wang, C.; Brown, S.; Veith, G. M.; Luo, H.; Liu, H.; Dai, S. A SuperacidCatalyzed Synthesis of Porous Membranes Based on Triazine Frameworks for $\mathrm{CO}_{2}$ Separation. J. Am. Chem. Soc. 2012, 134, 10478-10484.
(33) Huang, W.; Wang, Z. J.; Ma, B. C.; Ghasimi, S.; Gehrig, D.; Laquai, F.; Landfester, K.; Zhang, K. A. I. Hollow Nanoporous Covalent Triazine Frameworks: Via Acid Vapor-Assisted Solid Phase Synthesis for Enhanced Visible Light Photoactivity. J. Mater. Chem. A 2016, 4, 7555-7559.

(34) Kuecken, S.; Acharjya, A.; Zhi, L.; Schwarze, M.; Schomäcker, R.; Thomas, A. Fast Tuning of Covalent Triazine Frameworks for Photocatalytic Hydrogen Evolution. Chem. Commun. 2017, 53, 58545857.

(35) Sivakkumar, S. R.; Kim, D.-W. Polyaniline/Carbon Nanotube Composite Cathode for Rechargeable Lithium Polymer Batteries Assembled with Gel Polymer Electrolyte. J. Electrochem. Soc. 2007, 154, A134.

(36) Thompson, R.; Doggrell, S.; Hoberg, J. O. Potassium Channel Activators Based on the Benzopyran Substructure: Synthesis and Activity of the C-8 Substituent. Bioorg Med. Chem. 2003, 11, 16631668.

(37) Wallis, P. J.; Booth, K. J.; Patti, A. F.; Scott, J. L. Oxidative Coupling Revisited: Solvent-Free Heterogeneous and in Water. Green Chem. 2006, 8, 333.

(38) Gang, H.; Lee, D.; Choi, K. Y.; Kim, H. N.; Ryu, H.; Lee, D. S.; Kim, B. G. Development of High Performance Polyurethane Elastomers Using Vanillin-Based Green Polyol Chain Extender Originating from Lignocellulosic Biomass. ACS Sustainable Chem. Eng. 2017, 5, 4582-4588.

(39) Yang, G.; Zhang, Y.; Huang, Y.; Shakir, M. I.; Xu, Y. Incorporating Conjugated Carbonyl Compounds into Carbon Nanomaterials as Electrode Materials for Electrochemical Energy Storage. Phys. Chem. Chem. Phys. 2016, 18, 31361-31377.

(40) Balachandran, V.; Parimala, K. Vanillin and Isovanillin: Comparative Vibrational Spectroscopic Studies, Conformational Stability and NLO Properties by Density Functional Theory Calculations. Spectrochim. Acta, Part A 2012, 95, 354-368.

(41) Scheffer, J.; Wong, Y. F.; Patil, A. O.; Curtin, D. Y.; Paul, I. C. CPMAS (cross-polarization magic angle spinning) carbon-13 NMR Spectra of Quinones, Hydroquinones, and Their Complexes. Use of CMR To Follow a Reaction in the Solid State. J. Am. Chem. Soc. 1985, 107, 4898-4904.

(42) Birkett, H. E.; Cherryman, J. C.; Chippendale, A. M.; Evans, J. S. O.; Harris, R. K.; James, M.; King, I. J.; McPherson, G. J. Structural Investigations of Three Triazines: Solution-State NMR Studies of Internal Rotation and Structural Information from Solid-State NMR, plus a Full Structure Determination from Powder X-Ray Diffraction in One Case. Magn. Reson. Chem. 2003, 41, 324-336.

(43) Vujković, M.; Matović, L.; Krstić, J.; Stojmenović, M.; Đukić, A.; Babić, B.; Mentus, S. Mechanically Activated Carbonized Rayon Fibers as an Electrochemical Supercapacitor in Aqueous Solutions. Electrochim. Acta 2017, 245, 796-806.

(44) Dubal, D. P.; Ayyad, O.; Ruiz, V.; Gómez-Romero, P. Hybrid Energy Storage: The Merging of Battery and Supercapacitor Chemistries. Chem. Soc. Rev. 2015, 44, 1777-1790.

(45) Brousse, T.; Bélanger, D.; Long, J. W. To Be or Not To Be Pseudocapacitive? J. Electrochem. Soc. 2015, 162, A5185-A5189.

(46) Lukatskaya, M. R.; Dunn, B.; Gogotsi, Y. Multidimensional Materials and Device Architectures for Future Hybrid Energy Storage. Nat. Commun. 2016, 7, 1-13.

(47) Kim, S. K.; Kim, Y. K.; Lee, H.; Lee, S. B.; Park, H. S. Superior Pseudocapacitive Behavior of Confined Lignin Nanocrystals for Renewable Energy-Storage Materials. ChemSusChem 2014, 7, 10941101.

(48) Mukhopadhyay, A.; Jiao, Y.; Katahira, R.; Ciesielski, P. N.; Himmel, M.; Zhu, H. Heavy Metal-Free Tannin from Bark for Sustainable Energy Storage. Nano Lett. 2017, 17, 7897-7907.

(49) Patil, N.; Aqil, A.; Ouhib, F.; Admassie, S.; Inganäs, O.; Jérôme, C.; Detrembleur, C. Bioinspired Redox-Active Catechol-Bearing Polymers as Ultrarobust Organic Cathodes for Lithium Storage. Adv. Mater. 2017, 29, 1-9. 
(50) Gan, J. K.; Lim, Y. S.; Huang, N. M.; Lim, H. N. Boosting the Supercapacitive Properties of Polypyrrole with Chitosan and Hybrid Silver Nanoparticles/nanoclusters. RSC Adv. 2016, 6, 88925-88933.

(51) Kim, Y. T.; Mitani, T. Competitive Effect of Carbon Nanotubes Oxidation on Aqueous EDLC Performance: Balancing Hydrophilicity and Conductivity. J. Power Sources 2006, 158, 1517-1522.

(52) Ilic, I. K.; Perovic, M.; Liedel, C. The interplay of porosity, wettability, and redox activity asdetermining factors for lithiumorganic electrochemical energystorage using biomolecules. ChemSusChem 2020, DOI: 10.1002/cssc.201903156.

(53) Deblase, C. R.; Silberstein, K. E.; Truong, T. T.; Abruña, H. D.; Dichtel, W. R. B-Ketoenamine-Linked Covalent Organic Frameworks Capable of Pseudocapacitive Energy Storage. J. Am. Chem. Soc. 2013, $135,16821-16824$. 